Convergences francophones $5.2(2018): 92-105$

http://mrujs.mtroyal.ca/index.php/cf/index

\title{
Le voyage pittoresque et historique de l'Istrie et de la Dalmatie de Joseph Lavallée : l'enchâssement textuel tardif (1802) des dessins de Louis François Cassas réalisés en 1782
}

\author{
Dragan Bogojević \\ Université du Monténégro
}

\section{Introduction}

Le Musée des Beaux-Arts de Tours, détenteur d'un fonds Cassas (ce dernier est né à Azay-le-Ferron, localité proche de Tours), a récemment organisé, à l'initiative de sa conservatrice, une grande exposition consacrée à l'ample séquence italienne de l'œuvre de Cassas. Au début des années 1780, ce dernier a en effet été un artiste voyageur du Grand Tour italien traditionnel et aristocratique même si par ailleurs, sous la protection de Choiseul-Goufier son mécène d'alors, il a effectué un grand périple au Moyen Orient, de l'Egypte à la Syrie, qu'il a ponctué de dessins, qui, gravés, ont fait l'objet d'une publication partielle à la fin du XVIII siècle.

Les soixante-neuf dessins réalisés en 1782 lors du voyage de Cassas en Istrie et en Dalmatie s'inscrivent dans une dynamique originale d'extension et de redéfinition du Grand Tour. Avec le dégagement progressif des sites de Pompéi et d'Herculanum, le périple italien des antiquaires a connu une première et durable extension vers le sud : la région napolitaine, ses ruines antiques d'exception et son volcanisme fascinant constituent une polarisation sans précédent. On sait par ailleurs le rôle joué par Winkelmann, critique d'art et philosophe dans la promotion du retour à l'antique et dans le développement d'une esthétique néo-classique qui, de Dresde à Paris, de Londres à Saint-Pétersbourg, s'impose à toute l'Europe, en rupture avec les derniers échos du baroque et du rococo.

Mais avec Winkelmann, par-delà les cités romaines de Pompéi et Herculanum, ce sont bien les modèles esthétiques de la Grèce classique qui sont visés. Ces modèles architecturaux sur lesquels Winkelmann s'attarde avec, au sud de Naples, les temples de Paestum. La part grecque de la Sicile -la Grande Grèce d'Agrigente à Syracuse- s'avérera donc dans les années 1775-1780 la seconde extension d'un Grand Tour qui demeure plus que jamais le voyage des "antiquaires".

Avec Louis François Cassas, en 1782, le périple traditionnel des antiquaires connaît toutefois une ultime extension : la côte adriatique -l'Istrie et la Dalmatiedont les nombreux sites antiques exclusivement romains (il n'est pas ici de traces de colonies grecques) exigent désormais description, identification et inventaires. Cassas s'inscrit ici dans une dynamique déterminée par un contexte géopolitique précis : le mécénat de Joseph II et la politique d'expansion de l'Autriche vers le Sud-Est de l'Europe où les deux empires anciennement concurrents (Venise et l'Empire ottoman) sont en net recul. C'est en effet à partir d'une commande autrichienne (l'Autriche de Joseph II) que Cassas réalise soixante-neuf dessins destinés à être gravés à Vienne.

Ce n'est pourtant que vingt années plus tard et à Paris (1802, dans la France du Consulat) que ces dessins seront publiés, enchâssés dans le livre de Joseph 
Lavallée qui se propose de commenter, voire de compléter par ses propres évocations et descriptions les groupes de gravures qui scandent le développement du texte. Lavallée qui n'hésite pas de plus à se substituer à Cassas pour relater les péripéties d'un voyage terrestre et maritime complexe et risqué. Là encore le contexte qui préside à la prose de Lavallée doit être rappelé : alors que l'Autriche connaît défaite sur défaite, la France de Bonaparte, victorieuse en Italie s'intéresse à une région qui, quelques années plus tard, sous le nom de "provinces illyriennes" sera annexée à l'Empire français.

Dans son article intitulé «La publication du voyage pittoresque et historique de l'Istrie et de la Dalmatie » de L.F.Cassas et J. Lavallée dans le contexte de la politique napoléonienne en Illyrie Jean-Pierre Caillet s'est tout d'abord attaché à rappeler ce que furent le parcours et la carrière de Lavallée, « médiocre» littérateur qui « devait s'assurer une certaine notoriété par divers écrits plus ou moins directement à la gloire du régime alors en place » (Caillet 909). Il montre par ailleurs comment, dans le contexte particulier des ambitions régionales de Bonaparte, le luxueux ouvrage illustré des gravures de Cassas relaie tardivement les trois ouvrages qui, à la fin du XVIII ${ }^{\mathrm{e}}$ siècle, font autorité en matière de découverte et de connaissance de la région et de ses antiquités : « le Voyage d'Italie, de Dalmatie, de Grèce et du Levant » de J. Spon et G. Wheler (Lyon, 1678), « Ruins of the palace of the Emperor Diocletian at Spalato in Dalmatia » de R. Adam (Londres 1764), « le Voyage en Dalmatie » de l'Abbé G.B. Fortis (Berne, 1778). En outre, nous sommes témoins d'un intérêt accru pour le livre de Lavallée et de Cassas au XXe et XXIe siècle de la part des chercheurs et des historiens d'art croates qui s'en sont inspirés à différents niveaux. Ce sont surtout les aquarelles et les gravures de Cassas qui représentent un champ de recherche privilégié. Dans sa précieuse étude «Louis François Cassas i njegove slike Istre i Dalmacije » ${ }^{1}$ Kečkemet a eu l'intention de valoriser l'apport incontestable de Cassas au développement de courant classique et néo-classique en Europe dans plusieurs essors, en soulignant que « ... les descriptions de Cassas constituent des précieuses données dans le domaine de l'archéologie, de l'histoire de la culture et de l'ethnologie » (Kečkemet 200), en mettant en exergue le travail fait par Cassas qui, à son avis n'a nullement été reconnu comme il méritait. M. Rakić s'était directement inspirée du livre en question dans son article «Splitski spomenici u spisu Cassasa i Lavalléea » M. Rakić est absolument convaincue que le travail de Cassas «... apporte une richesse exceptionnelle des détails dans le domaine culturel et historique et des éléments importants pour l'ethnographie et l'iconographie maritime » (Rakić 72). En ce qui concerne le beau travail de Vesna Vlašić Jurić sur le palais de Dioclétien à Split, elle fait part notamment de gravures de Cassas, en décrivant pour l'essentiel l'aspect technique et architectural des gravures. T. Marasović et T. Alujević, font une étude approfondie sur la dimension architecturale du palais de Dioclétien et le livre de Lavallée et de Cassas figure dans la bibliographie des ouvrages référentiels, tandis que T. Marasović dans son article «O hramovima Dioklecianove palače $»^{2}$, souligne le fait que Cassas avait donné plusieurs gravures du palais, dont les plus impressionnants sont celles du mausolée

${ }^{1}$ Louis François Cassas et ses gravures d'Istrie et de Dalmatie.

${ }^{2}$ Sur les temples du palais de Dioclétien. 
et du temple rectangulaire (Marasović 95). La vision romantique de Cassas dans la présentation de l'aqueduc près de la rivière Jadro attire l'attention de A. Duplančić dans son article Salona na slici Carla Haasea ${ }^{3}$ (Duplančić 152). À juste titre, il est intéressent de noter que S. Bertoša et A. Giudici dans son article « Europski putnici u Istri od XV. do XIX stoljeća ${ }^{4}$, consacré aux voyageurs qui parcouraient l'Istrie et la Dalmatie n'ont même pas mentionné le livre de Lavallé et Cassas ! Peut-être, n'ont-ils pas voulu incorporer ces auteurs dans son corpus, vu que Lavallé n'a jamais mis le pied dans la région qu'il décrivait, puisque il s'appuyait dans son travail de récriture sur le journal (perdu) de Cassas?

Notre questionnement sera pour notre part résolument différent : nous tenterons de comprendre comment le récit-commentaire de Joseph Lavallée s'articule sur les gravures de Cassas et nous interrogerons plus précisément les écarts et décalages constitutifs d'un transfert culturel exceptionnel et à bien des égards paradoxal : du voyage de l'artiste français disposant de multiples protections et de la commande de l'Autriche de Joseph II à l'accompagnement "littéraire" du citoyen Lavallée et à la publication vingt ans plus tard dans une France révolutionnée qui répudie tout vandalisme et prétend hautement renouer avec le patrimoine classique.

\section{Les renvois des textes aux images}

La disposition des gravures de Cassas au sein du texte rédigé par Joseph Lavallée correspond globalement à l'itinéraire de l'artiste voyageur et, à partir de la deuxième partie, leur répartition par groupes séparés par des intervalles de texte invite à des parallèles et des mises en regard précises. Au premier regard textes et images semblent ainsi disposés en miroir. L'ample première partie, que Lavallée qualifie d'historique échappe pourtant à ce jeu de renvois. Elle ne comporte en effet aucune insertion de gravure et présente donc un fort degré d'autonomie que Lavallée se plait à souligner en clausule de cette partie. Il n'est pas encore là de collaboration avec l'œuvre gravée de Cassas : ce n'est qu'en incipit de la deuxième partie que Lavallée précise qu'il s'agit maintenant et maintenant seulement de «parcourir sur les pas du citoyen Cassas les côtes et les différentes villes qu'il a visitées » (Lavallée et Cassas 61).

À partir de la deuxième partie, le texte se présente comme un récit suivant l'ordre géographique et chronologique du voyage effectué vingt ans plus tôt. L'auteur narrateur — Joseph Lavallée - semble alors se substituer à Cassas qui n'a laissé qu'une suite ordonnée de gravures, traces iconiques de son périple. Alternent vues panoramiques de ruines, vues de " débris antiques », vues focalisées sur des monuments conservés dans leur intégralité ou dans leur structure (le palais de Dioclétien à Split notamment), vues panoramiques de rades, de villes et de bourgades. Interviennent aussi — et pour l'essentiel à la fin du voyage — des vues de cascades, lacs et torrents, éléments de paysages alpestres (en lisière de la Carniole - l'actuelle Slovénie) et au cœur de ces paysages, de quelques impressionnantes forteresses médiévales. L'élément humain paraît quasi absent -

\footnotetext{
${ }^{3}$ Salone sur le tableau de Carl Haase.

${ }^{4}$ Les voyageurs européens en Istrie et en Dalmatie du XVe au XIXe siècle
} 
ou réduit à de vagues silhouettes de taille extrêmement réduites en comparaison de la masse des monuments antiques et de l'ampleur des perspectives - dans ces gravures sans nul doute marquées par l'influence des Vues de Rome de Piranèse.

L'ambiguïté de l'ensemble texte/image est ici patente : l'œuvre gravée de Cassas tend à devenir une simple illustration du récit développé par Lavallée. Mais simultanément, et au sein même du récit qu'il développe, le narrateur se veut critique d'art et prétend, non sans lourdeur rhétorique, reconstituer et rendre sensible au lecteur ce qu'a été le point de vue de Cassas dessinateur. A propos d'une gravure représentant une impressionnante cataracte, Lavallée semble vouloir régir la vision du lecteur:

Le citoyen Cassas, voulant faire passer dans l'âme de ceux qui verraient ses dessins sans pouvoir les confronter avec la nature toutes les sensations qu'il avait éprouvées lui-même, après avoir admiré de près le sublime déchirement de ce fleuve sauvage, se recule, si j'ose m'exprimer ainsi, pour mieux saisir l'ensemble de cette scène, et connaître si les beautés de la masse répondent à la beauté des détails. Reculons avec lui; descendons le fleuve; mettons entre la cascade et nous cinq ou six cents toises : plaçons en idée, s'il nous est possible, le lecteur en face de cette superbe cataracte; et considérons avec le citoyen Cassas l'un des plus beaux spectacles du monde. (Lavallée et Cassas 105)

Mais le plus souvent les commentaires des gravures de Cassas s'orientent vers la méditation stéréotypée sur le temps destructeur et la vanité des ambitions humaines :

Ce fut donc là l'antique séjour des jeux et des plaisirs. Les bruyantes acclamations des peuples ont donc retenti sous ces murs. Aujourd'hui c'est l'asile du silence et de la mélancolie. Que de réflexions quand on y pénètre ! Qu'elle est éloquente la solitude dont on marche entouré ! On croit entendre la péroraison de l'histoire des empires. Quelle est la place que souillent ces reptiles ? Celle où s'asseyait Auguste. Où sont ces fleurs, ces myrtes, ces lauriers qui pendaient en festons sur ces Romains si fiers ? Des ronces dont la décrépite chevelure trahit la caducité des arceaux entrouverts, voilà les guirlandes appendues par le temps. Tout a disparu; et le gladiateur qui dessinait sa mort, et les Césars qui pesaient sur la terre; et les lions qui disputaient au criminel l'heure de son supplice; et l'histrion enrichi des vers de Sophocle et de Térence; et ces héros couverts de la pourpre triomphale; et ce peuple qui vendait aux jeux du cirque le souvenir de son esclavage; de ce monde de l'antiquité il ne reste rien, rien que des pierres. Ambitieux, errez parmi les ruines, elles vous prédiront le sort de vos tombeaux. (66)

\section{L'ambiguiité de la collaboration rédactionnelle}

Mais au-delà de la dimension critique et esthétique du texte de Lavallée, audelà des topoï que ce dernier développe de manière quasi continue, il importe d'analyser la présentation ambiguë de la collaboration du rédacteur — Lavallée- 
et du dessinateur - Cassas. Cette ambiguïté tient en fait à plusieurs écarts et décalages :

- l'unique signature de l'ouvrage est bien celle de Joseph Lavallée même si ce dernier ne se présente que comme "rédacteur";

- la totale absence de texte rédigé par Cassas - fût-ce même sous la forme d'une brève citation - n'empêche nullement qu'une centralité écrasante ne soit d'entrée de jeu conférée au dessinateur.

De prime abord, l'œuvre gravée de Cassas en effet s'impose. Le frontispice de l'ouvrage est une première gravure de Cassas représentant un amoncellement de débris et de trophées antiques, un bas-relief portant même le titre de l'ouvrage. Mais c'est sans nul doute la page de titre proprement dite, avec sa disposition et son jeu significatif sur les types de caractères d'imprimerie qui met en scène les équilibres subtils respectivement réservés à la part du "rédacteur" et à la part du dessinateur voyageur. Si dans cette page de titre l'auteur se présente modestement comme membre « de la Société philotechnique, de la Société libre des Sciences, Lettres et Arts de Paris, de celle d'Agriculture du département de Seine et Marne etc. ", Cassas est quant à lui introduit comme « peintre et architecte, auteur et éditeur du Voyage pittoresque de la Syrie, de la Phénicie, de la Palestine et de la Basse Egypte ».

Mais c'est avant tout l'ample liste des souscripteurs classée par ordre alphabétique qui, allusivement, confère un statut de médiateur d'exception à Joseph Lavallée "rédacteur". Ce dernier, par cette ample liste qui revêt en quelque sorte une valeur de dédicace, se met en scène comme artisan d'une reconnaissance assurée de son œuvre et de l'œuvre de Cassas qu'il lui attache. Cette reconnaissance est exhibée comme celle des plus hautes autorités de la République (les premiers souscripteurs nommés sont en effet les trois consuls - Bonaparte, Cambacérès et Lebrun -, les ministres, les généraux, le Sénat, le Corps législatif et le Tribunal) et les élites sociales et culturelles issues de la Révolution (dont de nombreux ingénieurs et architectes). Cassas est lui-même cité comme souscripteur pour cinquante exemplaires et diffuseur auprès de Julliot, peintre et architecte à Paris, Legrand, architecte des travaux publics, Limay, inspecteur des Ponts et Chaussées, Quatremère de Quincey, membre du Conseil général de la Seine, les frères Piranèse, chalcographes (c'est-à-dire graveurs sur cuivre).

L'examen de la première partie consacrée à l'histoire de l'Istrie et de la Dalmatie et dépourvue de toute insertion de gravure de Cassas, permet de dégager les objectifs propres à Lavallée : il s'agit avant tout pour ce dernier de comprendre la longue genèse de l'abaissement des populations de ces deux provinces, de la décadence qui, depuis l'époque romaine, a frappé la région de manière continue, réduisant ses peuples à une véritable sauvagerie :

Considérons sous ces deux points de vue l'Istrie et la Dalmatie qui présentent à l'observation la scène la plus curieuse : d'un côté le squelette de l'empire romain; de l'autre, et dans la Dalmatie surtout, un peuple nomade, et peut-être même redescendu par la dégradation à l'état sauvage; ici les traces fastueuses des maîtres du monde, là l'obscure indigence de quelques tribus ignorées, les colonnes décrépites des palais césariens, la hutte enfumée de l'Haiduck sans vertu; les arcs triomphaux de 
la victoire, les armes grossières du Morlaque sans milice; les restes majestueux des temples de Jupiter ${ }^{5}$, les informes chapelles du christianisme; les bains spacieux où la volupté romaine délassait les grâces et la beauté, la paille infecte où la Dalmatienne avilie repose loin de l'estime conjugale; enfin les ossements des arts et le corps difforme de l'ignorance. Tels sont les contrastes dont le rapprochement douloureux frappe à chaque pas le voyageur qui parcourt ces contrées. S'il étudie les ruines, elles lui rappellent des crimes et des erreurs; s'il étudie les habitants, il n'aperçoit que des souffrances et la stupidité. (Lavallée et Cassas 2)

L'exposé historique est tout d'abord largement consacré à la mainmise de Rome sur les deux provinces. Dans ce développement transparaissent nettement les préférences républicaines, anti-absolutistes et antichrétiennes de Lavallée: Auguste, le fondateur de l'empire et Constantin, converti et qui a fait du christianisme la religion de l'Etat sont vilipendés alors que Dioclétien, dont le palais de Split est l'objet de plusieurs gravures de Cassas, est réhabilité (il était généralement considéré comme l'un des plus farouches persécuteur des Chrétiens).

Le parcours historique postérieur aux "crimes des Césars" tente d'évaluer les retombées sur l'Istrie et la Dalmatie des grandes dynamiques catastrophiques de l'ère chrétienne : flux des "barbares" des grandes invasions, « troubles continuels de la cour de Byzance ... prétentions naissantes du catholicisme ... conquêtes des descendants de Mahomet ... longue et sanglante lutte du sacerdoce romain contre l'empire germanique » (Lavallée et Cassas 23) et enfin ambitions conquérantes de Venise. Selon Lavallée, cette succession des oppressions -auquel s'est ajouté "le croisement des races" a éteint l'ancestral esprit de résistance des Dalmates. Lavallée évoque en outre brièvement la présence des "manichéens" ${ }^{\prime \prime}$ qui ont suscité la haine des rois catholiques de Hongrie envahisseurs à leur tour de la Dalmatie. L'anarchie et la terreur ont surgi de tous les foyers simultanément et successivement en présence et suscité en retour des réactions tout aussi désordonnées et terribles :

Ici telle bourgade que l'inquisition vénitienne avait rendue déserte était repeuplée par des familles qui s'échappaient du despotisme hongrois; là l'on fuyait loin du joug des Musulmans vers des lieux que la férocité des Bulgares avait changé en solitude. Rois, nations, sultans, simples seigneurs, magistrats même, tout était dans ces climats un objet de terreur pour l'homme. (Lavallée et Cassas 29)

Issus de cette terreur omniprésente, les Uscoques —peuple de « transfuges », « brigands » ou « pirates »- ripostent avant de disparaitre à leur tour :

... je ne m'attacherai qu'à la Bosnie, cette partie du royaume de Servie qui fut démembré par les rois de Hongrie. Je n'en parlerai que parce qu'elle fut la perte par laquelle dans la suite les Turcs s'introduisirent en Dalmatie, et seulement pour arriver sans fatiguer le lecteur à lui donner quelque idée des

\footnotetext{
${ }^{5}$ Voir Figure 2 en fin d'article.

${ }^{6}$ Les Bogomils, terme que n'emploie pas Lavallée
} 
Uscoques, ce peuple de brigands qui, se formant tout à coup, ne descendant d'aucune nation, n'étant autre chose qu'un rassemblement de pirates et de malfaiteurs, fut néanmoins pendant seize lustres le plus redoutable et le plus implacable ennemi des musulmans et des Vénitiens; réunit sur sa tête tous les genres d'oppression, d'infortunes, de calamités et de supplices que la vindicte vénitienne et la barbarie mahométane purent inventer; et plutôt détruit que vaincu, plutôt massacré que soumis, disparut de la terre aussi rapidement, aussi silencieusement, pour ainsi dire, qu'il s'y était montré. (Lavallée et Cassas 37)

\section{La tutelle de la France révolutionnée : un avenir envisageable}

La pénétration des Turcs par la Bosnie a achevé la décadence de la Dalmatie qui a « ajouté la calamité des contrastes toujours si insupportables pour la multitude au fléau de l'esclavage » (Lavalée et Cassas 37). Lavallée dénonce en outre la «puérilité méprisable » de la politique des empereurs germaniques qui n'ont pas hésité à instrumentaliser contre Venise les Uscoques de plus en plus spécialisés dans le pillage maritime : «Les Vénitiens les aigrirent; l'église romaine préféra de les persécuter au devoir de les éclairer; la maison d'Autriche en fit les instruments de sa politique; les grands partagèrent avec eux; et quand le philosophe examine leur histoire, il ne voit pas que les Uscoques soient les seuls criminels » (44).

Lavallée met en fait en cause les politiques des Etats européens avant la Révolution française: ces politiques tyranniques à des degrés divers (Lavallée salue cependant l'aménagement de Trieste par l'impératrice Marie-Thérèse) ont permis et entretenu la pire des anarchies aux marches de l'Europe. Mais entre l'Istrie et la Dalmatie il est un autre peuple "sauvage" qui, s'il se fût uni avec les terribles Uscoques, eût été en mesure de constituer, après les contrées barbaresques d'Afrique du Nord, un deuxième « empire des pirates » en Méditerranée: il s'agit des Morlaques ${ }^{7}$. Ces derniers, qu'ils viennent des montagnes («bruns et farouches ») ou des plaines («blonds et doux ») sont un peuple foncièrement voleur. Mais cette propension au vol tient à une culture qui ignore le droit de propriété et a une dimension proprement communiste: «L'action du vol participe de ce désintéressement qui leur fait regarder tout entre eux comme à peu près commun » (47). Chez les Morlaques, cette pratique quasi coutumière du vol n'empêche nullement un sens inné de la générosité, de l'amitié et de l'hospitalité que Lavallée relie à leur qualité originaire de «peuple pasteur »: " L'on retrouve chez eux de profondes traces de la candeur des mœurs antiques, et surtout cette innocente liberté qui fut toujours le partage des peuples pasteurs » (48). Lavallée note chez les Morlaques l'omniprésence des magiciens et la pratique de rituels atroces de conjuration des vampires: ce sont là autant de traits que Lavallée identifie comme des signes majeurs d'un archaïsme qui a été également jusqu'à une période récente celui de la France. La tradition poétique orale des Morlaques, issue, selon Lavallée, de l'ancienne Illyrie mais également partie prenante des traditions germaniques, mérite enfin d'être prise en compte : inférieure mais comparable aux chants d'Ossian, elle doit être recueillie et étudiée :

\footnotetext{
${ }^{7}$ Voir Figure 1 en fin d'article.
} 
Nécessairement ces peuples ont eu leurs bardes; incontestable preuve que cette nation a tenu par son courage un rang parmi les nations barbares de la Germanie; je dis courage, car les peuples lâches n'ont point eu de poëtes. Il n'y a point de fêtes, d'assemblées chez les Morlaques où il ne se trouve un chanteur. Ces chansons, en idiôme illyrien, mais corrompu par le nombre de siècles qu'elles ont traversés avant d'arriver jusqu'à nous, sont l'histoire de quelques héros Slaves, ou la relation de quelque événement magique dont ils ignorent l'époque. Ce chant héroïque est grave, monotone et lugubre. L'instrument dont on l'accompagne n'est guère fait pour l'animer; c'est une méchante guitare monocorde dont le son est sourd et sans modulation. Cependant cette poésie n'est pas sans énergie; et sans avoir le sauvage éclat d'Ossian, elle en a quelquefois cette sorte de simplicité auguste dont le sentiment pénètre jusqu'à l'âme. Si un Morlaque voyage la nuit dans les montagnes, il est rare qu'il ne chante pas, et ces poèmes antiques sont toujours les chansons qu'il préfère. Une longue exclamation, ou plutôt un cri barbare et prolongé, précède chaque strophe. Il arrive souvent qu'il est entendu au loin par quelque Morlaque. (59-60)

Cette reconnaissance, au demeurant fort condescendante et à bien des égards restrictive, de la culture des Morlaques n'amoindrit en rien, dans le texte de Lavallée, le caractère négatif de la caractérisation des « habitants actuels de la Dalmatie (qui) présentent une grande matière aux réflexions du philosophe » (60). Ces populations semblent avoir été entraînées dans une irrémédiable spirale de décadence. Cette "moralité" des différents peuples d'Istrie et de Dalmatie transparaît dans tous leurs comportements et sont l'héritage d'une suite plus que millénaire de défaites, d'oppressions et de violences. La conjonction de tous les despotismes, de toutes les tyrannies, qu'elles soient occidentales ou orientales ont avili des populations qui semblent incapables de toute régénération :

Dans les Dalmatiens on retrouve encore toutes les petitesses des cours et d'Orient et de Rome. Partout, à leur air incertain, à leur politesse d'habitude, à leur marche ambiguë; à leur timidité circonflexe, on reconnaît des peuples dès longtemps ployés à être vaincus, et dont les défaites ont fréquemment changé de livrées; on reconnaît cet esprit d'intrigue, apanage des gouvernements débiles; ce costume de fausseté délateur de l'exil du civisme, cette souplesse innée qui court à la fortune individuelle à travers les débris de la prospérité publique; cette inquiétude tortueuse qui ne s'alimente que de brigues, que de mensonges obscurs, que de rivalités sourdes, que de religion affectée. (60)

Pour le Français Lavallée qui assume fièrement et, semble-t-il sans le moindre doute, l'héritage des Lumières et d'une Révolution fille des Lumières, l'Istrie et la Dalmatie n'ont d'avenir que dans la tutelle d'un pays engagé sur la voie de la liberté, de la justice et du progrès. Elles appellent la tutelle d'une "force majeure". La France révolutionnée n'est certes pas nommée mais tout laisse à penser que l'intervention victorieuse des armées de la République en Italie du nord permet d'envisager une extension aux côtes dalmates. Ce qui, avec l'Empire, ne tardera pas à se faire. 
Dans le long développement historique et politique de son texte, Lavallée continue certes à se réclamer de Cassas voyageur. Ainsi avant d'amorcer un long exposé sur la "situation géographique et l'histoire politique des deux provinces » Lavallée attribue au " citoyen Cassas » — mais à titre d'hypothèse seulement — une méditation désolée sur la décadence des deux provinces : «Telles sont les réflexions qui durent nécessairement affliger le citoyen Cassas au sein des plaisirs qu'offraient au génie du peintre la dignité et le piquant aspect des ruines » (2).

Mais à la fin de son ouvrage, Lavallée reconnaît avoir dû combler un manque : le « citoyen Cassas » a, conformément aux objectifs initiaux de son voyage, observé et dessiné les nombreux sites antiques jusqu'alors peu connus ainsi que les phénomènes naturels les plus « pittoresques ». Mais selon Lavallée, Cassas a ignoré la nécessité d'observer les peuples et leurs mœurs :

Il eût été à souhaiter peut-être que le citoyen Cassas eût joint aux qualités si recommandables de l'artiste l'esprit d'observation et des connaissances politiques : le lecteur, à la satisfaction d'apprendre en quel état se trouvent en ces lieux les vestiges de l'antiquité, eût ajouté le plaisir de connaître plus à fond les mœurs, les habitudes, les lois, et l'origine des peuples divers qui vivent sous ces climats. Mais ce n'était point-là le projet ni le but de Cassas: il lui a suffi de remplir celui qu'il s'était proposé et l'on a vu qu'il s'en est acquitté. (155)

\section{Conclusion}

Editeur des gravures de Cassas, rédacteur du texte qui accompagne les soixante-neuf gravures de l'artiste voyageur, Lavallée se présente donc comme le médiateur qui, par-delà la Révolution, est en mesure de pallier une faille proprement politique de l'artiste : son incapacité à rendre compte de la décrépitude des populations sinon rencontrées du moins croisées. Lavallée sous-entend que cette incapacité est datée et liée aux contraintes mêmes de la commande de l'empereur Joseph II, commande dont Cassas, qui n'était pas encore le « citoyen de Cassas », a été, en artiste d'ancien régime, bénéficiaire:

Il est heureux pour ma patrie que les circonstances aient voulu qu'un voyage, dont l'idée fut due à l'empereur Joseph II, et dont les résultats devaient lui appartenir, ait entièrement tourné au bénéfice de la République française. C'est ainsi que la pensée d'un prince philosophe, quel que soit le cours que prennent les événements, n'est jamais perdue pour l'humanité (56)

Malgré son indifférence et sa cécité à l'égard des peuples des pays visités, qui n'apparaissent pas ou très peu dans les dessins réalisés, le legs artistique de Cassas mérite d'être reçu et promu par la jeune République française. Mais ce legs doit être actualisé, complété et enrichi par l'adjonction d'un texte proprement politique et philosophique censé lui permettre d'intégrer le patrimoine d'une République qui, loin de tout vandalisme révolutionnaire, revendique l'héritage artistique de l'humanité toute entière. Et c'est là le rôle que s'assigne Lavallée qui 
s'affirme avant tout comme agent efficace d'une " assimilation critique » des legs artistiques de tous les temps antérieurs à la Révolution.

Le Voyage historique et pittoresque de l'Istrie et de la Dalmatie est ici révélateur des ambitions patrimoniales des élites sociales et culturelles nouvelles, issues de la Révolution. Ces élites dont la longue liste des souscripteurs publiée en tête de l'ouvrage exhibe les noms et qualités.

\section{Bibliographie}

Bertoša, Slaven et Antonio Giudici. «Evropski putnici u Istri od XV. do XIX.

Stoljeća. » Tabula : časopis Filozofskog fakulteta, Sveučilište Jurja

Dobrile u Puli, vol. 11, 2013, pp.7-35. https://hrcak.srce.hr/116819. Consulté le 12 avril 2018.

Caillet, Jean-Pirre. La publication du voyages pittoresque et historique de l'Istrie et de la Dalmatie de L.-F. Cassas et J. Lavallée dans le contexte de la politique napoléonienne en Illyrie. HAZU: Zagreb, 2010.

Cassas, Louis-François. « Vue de Pirano. » 1782. $<$ https://commons.wikimedia.org/wiki/File:Louis-Fran\%C3\%A7ois_Cassas_Piran_(2).jpg>

Duplančić, Arsen. « Salona na slici Carla Haasea. » Tusculum: časopis za solinske teme, vol.9, no.1, 2016, pp.147-171. https://hrcak.srce.hr/169767.

Consulté le 12 avril 2018.

Fortis, Abbé. Voyage en Dalmatie. Berne, Société Typographique, 1778.

Kečkemet, Duško. « Louis François Cassas i njegove slike Istre i Dalmacije. » Rad JAZU, knj. 379, Zagreb, 1978, pp.7-200.

Lavallée, Joseph et Louis-François Cassas. Voyage pittoresque et historique de l'Istrie et de la Dalmatie. Paris : Pierre Didot l'aîné, 1802.

Marasović, Tomislav. "O hramovima Dioklecijanove palače. » Prilozi povijesti umjetnosti u Dalmaciji, vol. 35, no.1, pp.89-101.

https://hrcak.srce.hr/114575. 1995. Consulté le 12 avril 2018.

Marasović, Tomislav et Tomislav Alujević. « Dioklecijanov stan u splitskoj palači." Prostor: znanstveni časopis za arhitekturu i urbanizam, vol.15, no.2 (34), pp.154-179, 2007. https://hrcak.srce.hr/18516. Consulté le 12 avril 2018.

Rakić, Marina. « Splitski spomenici u putopisu Cassasa i Lavalléea. » Kulturna baština, vol.36, pp.59-82, 2010. https://hrcak.srce.hr/65370?lang=en. Consulté le 12 avril 2018.

Sočivica. London Magazine: Or, Gentleman's Monthly Intelligencer, vol. XLVIII. C. Ackers, p. 50, 1779.

$<$ https://commons.wikimedia.org/wiki/File:So\%C4\%8Divica_(1779).jpg>

Vlašić Jurić Vesna. « Dioklecijanova palača na prikazima u grafičkoj zbirci nacionalne i sveučilišne knjižnice u Zagrebu. » Kroatologija časopis za hrvatsku kulturu, vol.2, no.1, pp.216-216, 2011. https://hrcak.srce.hr/75502. Consulté le 12 avril 2018.

«Vue d'une partie du Temple de Jupiter, du côté de la porte d'entrée. » Voyage pittoresque d'Istrie et de la Dalmatie. Joseph Lavallée et Louis-François Cassas, 18002. Biblioteca nazionale Marciana - Venezia - IT-VE0049. Identifiant : GEO00 18868 
Winkelmann, Johann Joachim. Remarques sur l'architecture des Anciens (Ûber die Baukunst der Alten) suivies d'un compte-rendu sur les temples de Paestum. Trad. Jansen Hendrik. Paris : Barrois aîné, 1783. ark:/12148/bpt6k10412698. Consulté le 12 avril 2018.

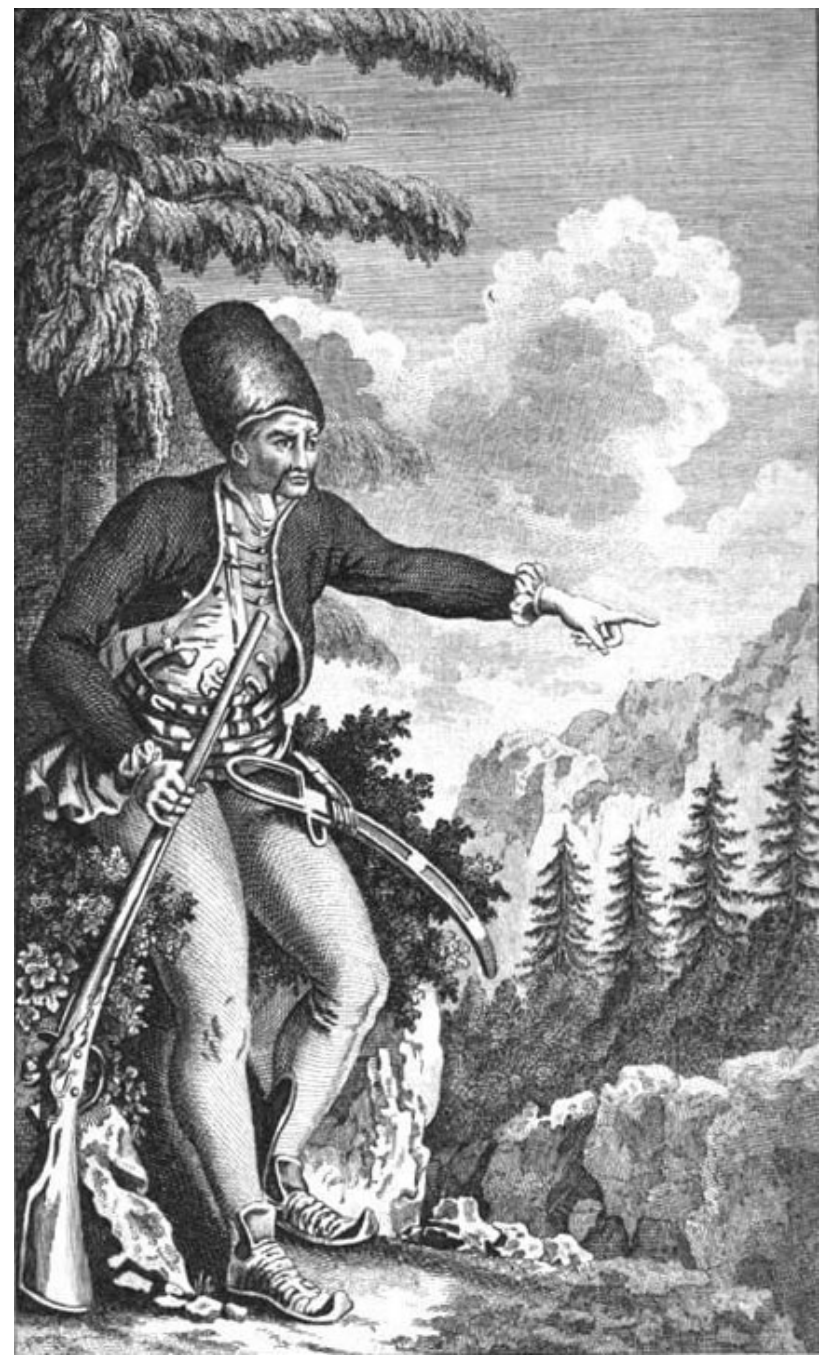

Fig.1 « Sočivica ». Tableau représentant un Morlaque. 
Convergences francophones 5.2 (2018) : 92-105

http://mrujs.mtroyal.ca/index.php/cf/index

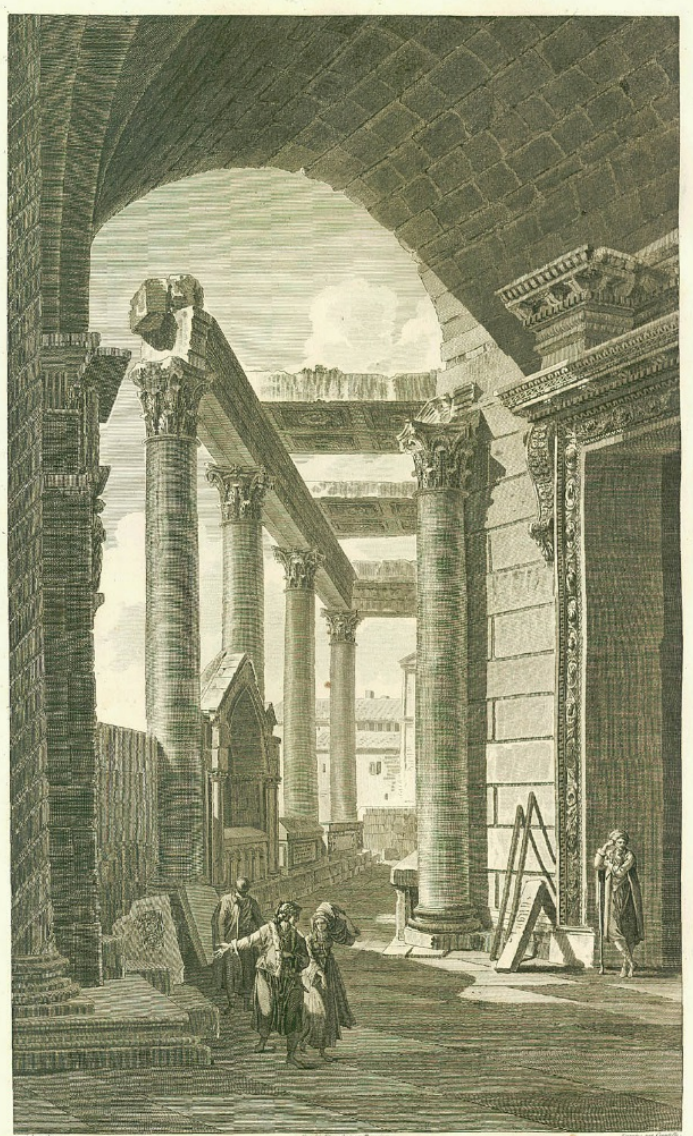

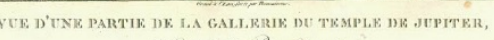

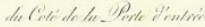

Fig. 2. «Vue d'une partie du Temple du Jupiter, du côté de la porte d'entrée » 


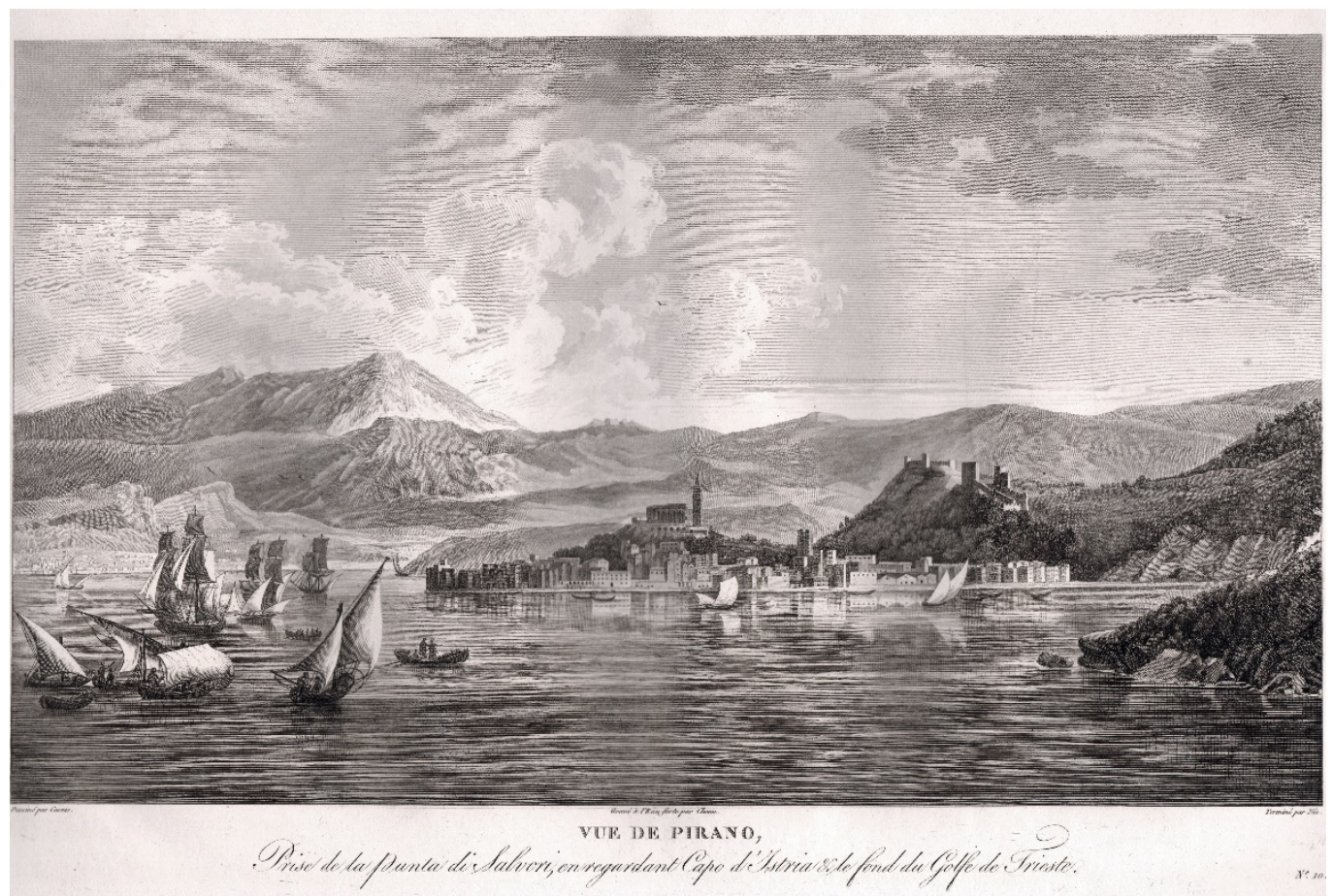

Fig.3 « Vue de Pirano » par Louis-François Cassas 